\title{
Warfarina versus acenocumarol en alcanzar niveles terapéuticos en una población ambulatoria
}

\author{
Raúl Romero L1, Pablo Vargas $\mathbf{M}^{2}$, Víctor Letelier A². $^{2}$. \\ 1 Unidad de Paciente Crítico Adultos, Hospital Regional de Iquique. \\ 2 Interno Medicina, Universidad del Mar, Sede Iquique
}

\section{Resumen}

Introducción: La anticoagulación constituye una terapia farmacológica habitual en la práctica clínica diaria. En Chile, los ACO disponibles y utilizados son warfarina y acenocumarol, no existiendo mayores experiencias nacionales documentadas sobre el mayor beneficio de un fármaco en particular.

Objetivo: Analizar y comparar la eficacia terapéutica de warfarina y acenocumarol en una población ambulatoria.

Metodología: Estudio retrospectivo, longitudinal. Se analizó 188 pacientes que estuvieron en tratamiento con acenocumarol durante más de un año, y que luego fueron cambiados a warfarina. Se registró: sexo, edad, efectos adversos, diagnóstico y justificación de inicio de ACO. Se obtuvo el promedio del International Normalizad Ratio (INR) de los últimos 3 meses de tratamiento con acenocumarol. Luego, se sustituyó por warfarina, obteniendo luego de un año de tratamiento, el INR promedio de los últimos 3 meses. Los pacientes se agruparon en tres grupos: Bajo rango terapéutico (INR<2.0), en rango terapéutico (INR=2.0-3.0), sobre rango terapéutico (INR>3.0).

Resultados: En los pacientes con acenocumarol, se observó 67 (35,64\%) bajo rango terapéutico; 91 (48,4\%) en rango terapéutico; y $30(15,96 \%)$ sobre rango terapéutico. Luego del cambio a warfarina, $76(40,43 \%)$ bajo rango terapéutico; 95 (50,53\%) en rango terapéutico; y 17 (9,04\%) sobre rango terapéutico, diferencias no significativas. Bajo el efecto de ambos fármacos no se registraron hemorragias mayores y no hubo diferencia significativa en hemorragias menores.

Discusión: La eficacia terapéutica fue similar con ambos fármacos. A pesar de que con acenocumarol se obtuvo mayor porcentaje de pacientes sobre rango terapéutico, no se observaron complicaciones mayores en el periodo de seguimiento.

Palabras Clave: Anticoagulación oral, warfarina, acenocumarol, rango terapéutico.

\section{Comparison of acenocumarol vs. warfarin in achieving oral anticoagulation therapeutic levels in ambulatory patients}

Background: oral anticoagulation is frequently needed in clinical practice. Warfarin and acenocumarol are available in Chile for this purpose. Locally, there is no evidence favoring one over the other.

Aim: To compare the efficacy of warfarin and acenocumarol in an ambulatory population.

Method: A retrospective study compared data on 188 patients with over 1 year of treatment with acenocumarol, before and after being switched over to treatment with warfarin. Demographic data, adverse effects, diagnosis and indication for oral anticoagulation were record. INRs obtained in the last 3 months of treatment with each agent were compared. Patients were classified in 3 groups: insufficient level (INR < 2.0), adequate level (INR 2.0 - 3.0) and high level (INR > 3.0) of anticoagulation.

Correspondencia: Raúl Romero Lamas

Unidad de Paciente Crítico Adultos

Hospital Regional de Iquique

Correo Eléctronico: raulromerolamas@gmail.com 
Results: With acenocumarol, low level INR was present in 35.6\%, adequate INR in 48.4\% and high INR in $15.9 \%$ of subjects. After switching to warfarin, corresponding levels in each group were $40.4 \%, 50.3 \%$ and $9 \%$ (NS). There were no serious bleeding episodes in either group; minor hemorrhages occurred with similar frequency in both groups.

Conclusion: There was similar clinical efficacy of oral anticoagulation with acenocumarol compared to warfarin. The slightly higher percentage of acenocumarol treated patients exhibiting a high IRN level did not result in increased risk of hemorrhage.

Keywords: Oral anticoagulation, warfarin, acenocumarol, therapeutic range.

Recibido el 24 de agosto de 2009, aceptado el 10 de noviembre de 2009

Rev Chil Cardiol 2009; 28: 375-379

\section{Introducción}

La anticoagulación oral constituye una terapia farmacológica habitual en la práctica clínica diaria, fundamentalmente en pacientes con patología cardiovascular y tromboembólica1, 2. Idealmente, esta terapia debe contar con que el efecto-dosis y la dosis individual de mantenimiento sean predecibles, entre otras condiciones. Sin embargo, actualmente ningún anticoagulante oral (ACO) las reúne 3-5.

Dentro de los fármacos anticoagulantes orales (ACO) disponibles, los más usados en la actualidad son los derivados cumarínicos 6 . Éstos ACO son antagonistas de la vitamina $\mathrm{K}$, cuyo efecto anticoagulante se debe a la inhibición de su enzima epóxido reductasa, interfiriendo en la gammacarboxilación de los residuos de glutamato en los factores II, V, VII y IX, además de inhibir la carboxilación de la proteína C y $S$ 1, 4-8.

La literatura muestra a que el ACO más usado es warfarina, cuya ventaja aparentemente es una vida media más larga y mayor estabilidad en mantener los niveles terapéuticos, lo que aún es cuestionado4, 9-11.

En Chile, los ACO disponibles y utilizados son warfarina y acenocumarol, no existiendo mayores experiencias nacionales documentadas sobre el mayor beneficio de un fármaco en particular.

Por esto, resulta interesante analizar y comparar la eficacia terapéutica de ambos fármacos en una población ambulatoria de nuestro hospital.

\section{Pacientes y Método}

Estudio retrospectivo, observacional de corte Ion gitudinal, en el cual se analizó a un grupo de pacientes controlados en el policlínico de anticoagulantes del Hospital Regional de Iquique, Chile, que estando en tratamiento con Acenocumarol fueron cambiados a tratamiento con Warfarina.

El universo estuvo compuesto por 233 pacientes, correspondientes al $50 \%$ de pacientes controlados en el policlínico.

Los criterios de inclusión fueron: Pacientes en tratamiento con acenocumarol durante más de un año, cambiados a warfarina.

Como criterios de exclusión se consideró: Pacientes en tratamiento con ACO por valvulopatías, abandono de control y/o tratamiento, pacientes con menos de un año de tratamiento con warfarina luego del cambio, pacientes que completaron el tratamiento ACO. De esta forma, se obtuvo el grupo estudio, conformado por 188 pacientes. Se registró en base a las fichas clínicas: Sexo, Edad, efectos adversos (hemorragias mayores y menores, definidas las primeras como aquellas que requirieron hospitalización y/o transfusiones) ${ }^{11}$, diagnóstico y justificación de inicio de ACO.

En una primera instancia, se obtuvo el promedio de los valores del International Normalizad Ratio (INR) de los últimos 3 meses de tratamiento con acenocumarol.

Posterior a eso, se sustituyó este fármaco por warfarina, obteniendo nuevamente, luego de un año de tratamiento, el promedio de los valores del INR de los últimos 3 meses de control.

Durante el tratamiento con acenocumarol, los pacientes fueron controlados cada 25 días como 
promedio, mientras que durante el tratamiento con warfarina se controló a los pacientes estables cada 52 días como promedio.

Los resultados obtenidos se agruparon en tres grupos, de acuerdo a las normas de la AHA \& ACC1: Bajo rango terapéutico (INR<2.0), en rango terapéutico (INR: 2.0-3.0), sobre rango terapéutico (>3.0.).

Posteriormente, se procedió a comparar, los resultados obtenidos del tratamiento con acenocumarol versus los obtenidos del tratamiento con warfarina, de acuerdo a cada grupo de valores promedio de INR. (Figura $\mathrm{n}^{\circ}$ 1).

Para el análisis estadístico se utilizó el programa SPSS11,5. Las variables cuantitativas se expresaron como media y desviación estándar, mientras que las variables cualitativas se expresaron porcentualmente. Se utilizó la prueba $T$ de Student.

Se consideró significativamente estadístico un valor de $p<0,05$.

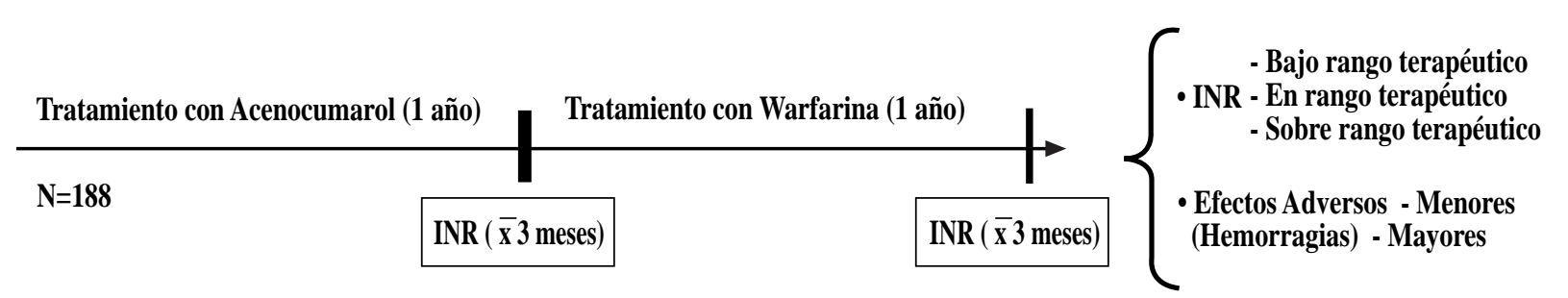

Figura 1: Diseño metodológico.

\section{Resultados}

El grupo estudio estuvo conformado por 188 pacientes; 96 hombres $(51,06 \%)$ y 92 mujeres $(48,94 \% \%)$, con un promedio de edad de 65,6 con desviación estándar de 12,9 años.
Respecto al inicio del ACO: 145 (77,13\%) pacientes iniciaron tratamiento por arritmias supraventriculares, $33(17,55 \%)$ por causas tromboembólicas y 10 (5,32\%) por otras causas. (Figura $\mathrm{n}^{\circ} 2$ ).

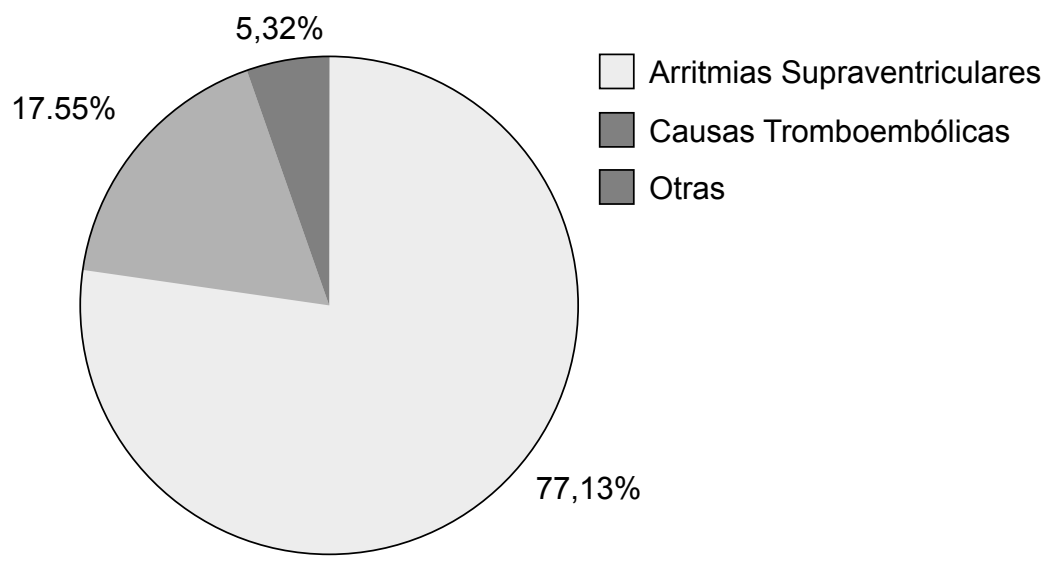

Figura 2. Causas de indicación de anticoagulación oral 
En el control de INR realizado a los pacientes estando en tratamiento con acenocumarol, se observó a 67 pacientes $(35,64 \%)$ bajo el rango terapéutico; $91(48,4 \%)$ en rango terapéutico; y 30 $(15,96 \%)$ sobre rango terapéutico.

Mientras que luego del cambio a warfarina, se observó a 76 pacientes $(40,43 \%)$ bajo rango terapéutico; 95 (50,53\%) en rango terapéutico; y 17 $(9,04 \%)$ sobre rango terapéutico.

Las diferencias observadas en los tres grupos con el cambio de medicamento no fueron estadísticamente significativas $(p>0,05)$. (Tabla $\left.n^{0} 1\right)$.

Tabla 1: Grupos según INR en tratamiento con acenocumarol warfarina

\begin{tabular}{cccc}
\hline INR & Acenocumarol & Warfarina & p value $(95 \% \mathrm{Cl})$ \\
\hline$<2.0$ & $67(35,64 \%)$ & $76(40,43 \%)$ & 0.189 \\
$2,0-3,0$ & $91(48,4 \%)$ & $95(50,53 \%)$ & 0,485 \\
$>3,0$ & $30(15,96 \%)$ & $17(9,04 \%)$ & 0,389 \\
\hline
\end{tabular}

En cuanto a las complicaciones observadas durante el tiempo con acenocumarol y posteriormente con warfarina, bajo el efecto de ambos fármacos no se observaron en el período de seguimiento hemorragias mayores y no hubo diferencia significativa en el porcentaje de hemorragias menores $(p>0,05)$, las que se presentaron en 8 pacientes $(4,26 \%)$ y 7 pacientes $(3,72 \%)$ para el grupo en control con acenocumarol y warfarina respectivamente.

\section{Discusión}

Durante el tratamiento ACO con ambos fármacos no hubo diferencias significativas en cuanto al número de pacientes que logró alcanzar niveles terapéuticos, lo que también se observó en una serie de 284 pacientes publicada por Pattacini et al13. Sin embargo, cabe señalar que dicho trabajo comparó a dos grupos con cada fármaco en paralelo, no existiendo en la literatura revisada trabajos que analicen la eficacia del tratamiento con ACO en un mismo grupo de estudio.

Respecto a los efectos adversos, tampoco hubo diferencias significativas en el desarrollo de hemorragias mayores ni menores, lo que no se correlaciona con lo expuesto en la literatura, en series como la de Rodríguez et al que registró un $32 \%$ y $11 \%$ de hemorragias con el uso de warfarina y acenocumarol respectivamente ${ }^{14}$.

En función de esto, podría ser cuestionable la forma en que se consignan los eventos hemorrágicos mayores y/o menores en la consulta de control del paciente en tratamiento con ACO, siendo planteable y necesario el diseño de un cuestionario validado para el registro de los mismos.

Cabe destacar que el grupo de profesionales que manejó a estos pacientes tenía una experiencia mínima de 5 años tratando enfermos con anticoagulantes.

Por otro lado, cabe señalar que pese a los niveles terapéuticos aceptables en nuestro grupo de estudio, aún falta mucho que decir respecto a cómo mejorar la terapia anticoagulante, desde potenciar métodos innovadores, como el automonitoreo en pacientes seleccionados $5,9,15$, hasta el desarrollo de ACO más eficaces y que se acerquen al cumplimiento de las condiciones ideales para este tipo de medicamentos ${ }^{16-19}$.

Finalmente, podemos concluir que en este estudio la eficacia terapéutica fue igual con ambos fármacos, y a pesar que con acenocumarol se obtuvo mayor porcentaje de pacientes sobre rango terapéutico, no se observaron complicaciones mayores en el período de seguimiento. 


\section{Referencias}

1. HIRSH J, FUSTER V, ANSELL J, HALPERIN JL; American Heart Association / American College of Cardiology Foundation guide to warfarin therapy. Circulation 2003; 107: 1692-1711.

2. ANSELL J, HIRSH J, POLLER L, BUSSEY H, JACOBSON A, HYLEK E. The Pharmacology and Management of the Vitamin K Antagonists: The Seventh ACCP Conference on Antithrombotic and Thrombolytic Therapy. Chest 2004; 126: 204S-233S

3. FREIXA R, BLANCH P, IBERNÓN J, PADRÓ J, DELSO J, SOBREPERA J, et al. Identificación de factores responsables de anticoagulación oral excesiva en pacientes ambulatorios con cardiopatía. Rev Esp Cardiol. 2003; 56: 65-72.

4. HIRSH J, DALEN JE, ANDERSON D, POLLER L, BUSSEY $H$, ANSELL $J$, et al. Oral anticoagulants: mechanism of action, clinical effectiveness, and optimal therapeutic range. Chest 2001; 119: 8-21.

5. FITZMAURICE D, MACHIN S. Recommendations for patients undertaking self management of oral anticoagulation. BMJ 2001; 323: 985-9.

6. BRUMMEL K, PARADIS S, BRANDA R, MANN KG. Oral anticoagulation thresholds. Circulation 2001; 104: 2311-2317.

7. CHOONARA I, MALIA R, HAYNES B, HAY CR, CHOLERTON $S$, BRECKENRIDGE AM, et al. The relationship between inhibition of vitamin $\mathrm{K}$ 1,2,3-epoxide reductase and reduction of clotting factor activity with warfarin. $\mathrm{Br} J$ Clin Pharmacol 1988; 25:1-7

8. NAVARRO RJ, VALENCIA SJS, ABUNDES VA, BETANCOURT HL. Anticoagulantes. Rev Mex Cardiol 2000; 11: 304-313.

9. HENEGHAN C, ALONSO-COELLO P, GARCIA-ALAMINO J, PERERA R, MEATS E, GLASZIOU P. Self-monitoring of oral anticoagulation: a systematic review and meta-analysis. The Lancet 2006; 367:404-11.
10. CÓliCA D, WAINBERG G, BARRAGÁN A. Beneficio de la anticoagulación oral con Acenocumarol en pacientes con fibrilación auricular crónica y su importancia en la práctica clínica. Rev del HPC 2007; 10: 36-41.

11. KEARON C, GINSBERG J, KOVACS M, ANDERSON DR, WELLS P, JULIAN JA, et al. Comparison of low-intensity warfarin therapy with conventional-intensity warfarin therapy for long-term prevention of recurrent venous thromboembolism. N Engl J Med. 2003; 349: 631-639.

12. HYLEK EM, EVANS-MOLINA C, SHEA C, HENAULT LE, REGAN S. Major Hemorrhage and Tolerability of Warfarin in the First Year of Therapy Among Elderly Patients With Atrial Fibrillation. Circulation 2007; 115: 2689-2696.

13. PATTACINI C, MANOTTI C, PINI M, QUINTAVALLA R, DETTORI AG. A comparative study on the quality of oral anticoagulant therapy (warfarin versus acenocoumarol). Thromb Haemost. 1994; 71:188-91.

14. AMIÁN A, RODRÍGUEZ JN, MUÑIZ R, DIÉGUEZ JC, MORENO MV, QUESADA JA, et al. Comparative study of the stability of oral anticoagulant treatments (warfarin vs acenocoumarol). Sangre (Barc). 1996; 41: 9-11.

15. DOUKETIS J. Patient self-monitoring of oral anticoagulant therapy. Potential benefits and implications for clinical practice. Am J Cardiovasc Drugs 2001;1: 245-51.

16. WEITZ J. New anticoagulants for treatment of venous thromboembolism. Circulation 2004; 110: I19-I26.

17. SPORTIF Executive Committee for the SPORTIF $V$ Investigators. Ximelagatran vs. warfarin for stroke prevention in patients with nonvalvular atrial fibrillation: a randomized trial. JAMA. 2005; 293: 690-698.

18. EIKELBOOM J, WEITZ J. A replacement for warfarin: The search continues. Circulation 2007; 116:131-133.

19. MONTAGNA R, ASENJO R, MORRIS R, ORTIZ M, CERECEDA M. Avances en la prevención de eventos tromboembólicos en pacientes con fibrilación auricular. Rev Méd Chile 2007; 135: 1048-1055. 